\title{
Espécie nova de Asemostera Simon e primeiro registro de A. latithorax (Keyserling) para o Rio Grande do Sul, Brasil (Araneae, Linyphiidae) ${ }^{1}$
}

\author{
Everton N. L. Rodrigues ${ }^{2}$ \\ 1 Trabalho realizado no Laboratório de Aracnologia, Museu de Ciências Naturais, Fundação Zoobotânica do Rio Grande do \\ Sul. Rua Dr Salvador França 1427, 90690-000 Porto Alegre, Rio Grande do Sul, Brasil. \\ 2 Programa de Pós-Graduação em Biologia Animal, Departamento de Zoologia, Instituto de Biociências, Universidade Federal \\ do Rio Grande do Sul. Avenida Bento Gonçalves 9500, Bloco IV, Prédio 43435, 91501-970 Porto Alegre, Rio Grande do Sul, \\ Brasil. Bolsista do CNPq. E-mail: enlrodrigues@yahoo.com.br
}

\begin{abstract}
New species of Asemostera Simon and first record of A. latithorax (Keyserling) from Rio Grande do Sul, Brazil (Araneae, Linyphiidae). Asemostera tacuapi sp. nov. from Rio Grande do Sul state is described and illustrated and A. latithorax (Keyserling, 1886) is newly recorded for that state.

KEY WORDS. Erigoninae; Neotropical; new record; spiders; taxonomy.
\end{abstract}

RESUMO. Asemostera tacuapi sp. nov. do estado do Rio Grande do Sul é descrita e ilustrada e A. latithorax (Keyserling, 1886) é registrada pela primeira vez para esse estado.

PALAVRAS-CHAVE. Aranhas; Erigoninae; Neotropical; nova ocorrência; taxonomia.

O gênero Asemostera Simon, 1898 foi proposto por SIMON (1898) designando como espécie-tipo A. latithorax (Keyserling, 1886). O gênero manteve-se monotípico até a recente revisão da subfamília Erigoninae na região Neotropical, realizada por Miller (2007), o qual incluiu como sinônimos quatro gêneros monotípicos: Pelidida Simon, 1898, Caleurema Millidge, 1991, Asemonetes Millidge, 1991 e Ochronetria Millidge, 1991. Atualmente o elenco de Asemostera é composto por sete espécies neotropicais: A. arcana (Millidge, 1991), A. daedalus Miller, 2007, A. enkidu Miller, 2007, A. involuta (Millidge, 1991), A. janetae Miller, 2007, A. latithorax e A. pallida (Millidge, 1991). Somente $A$. latithorax é registrada para o Brasil e nenhuma para o Rio Grande do Sul (Miller 2007).

Essas aranhas são caracterizadas pelo cefalotórax, aproximadamente, mais largo do que longo, com uma porção de cerdas entre os olhos; esterno fortemente truncado; palpo do macho com proeminente membrana embólica, projetando-se distalmente, êmbolo e processo radical anterior filiformes; fêmea com tarso do palpo longo e intumescido, epígino com trajetória dos ductos copulatórios complexa e átrio saliente ou simples com curtos ductos copulatórios (Miller 2007).

Neste trabalho, A. latithorax é registrada pela primeira vez para o Rio Grande do Sul e é descrita uma espécie nova, cujo material está depositado na coleção aracnológica do Museu de Ciências Naturais, Fundação Zoobotânica do Rio Grande do Sul, Porto Alegre (MCN; E.H. Buckup). A posição da tricobótria no metatarso da perna I (TmI) foi calculada conforme Millidge (1980). Descrições seguem Miller (2007). O estudo das estruturas internas da genitália da fêmea foi realizado mergulhando o epígino em ácido láctico 85\%. Medidas são fornecidas em milímetros (mm). Abreviaturas utilizadas para os olhos: (OLA) olhos laterais anteriores, (OLP) olhos laterais posteriores, (OMA) olhos médios anteriores e (OMP) olhos médios posteriores.

\section{Asemostera tacuapi sp. nov. \\ Figs 1-9}

Material-tipo. Holótipo macho macho, Brasil, Rio Grande do Sul: Triunfo (Parque Copesul de Proteção Ambiental), 15.III.2007, M.A.L. Marques leg. (MCN 43254). Parátipo fêmea, mesma localidade do holótipo, 12.XII.2006, A. Barcellos \& R. Ott leg. (MCN 42741); macho, fêmea, mesma localidade e coletor do holótipo, 24.XI.1999 (MCN 31633).

Etimologia. O nome específico provém do guarani e refere-se a uma espécie de canudo (bomba) de taquara, utilizada pelos índios para sorver o chimarrão (mate) em uma cabaça (cuia).

Diagnose. O palpo do macho de Asemostera tacuapi sp. nov. assemelha-se ao de A. enkidu Miller, 2007 (Miller 2007, figs 127e, f, i) por apresentar em comum o rádix não encobrindo o supratégulo e pelo processo radical anterior não tão longo e seguindo o mesmo percurso do êmbolo (Fig. 4), mas difere pela membrana embólica distalmente mais curta, paracímbio afilado, apófise tibial retrolateral arredondada, intumescida distalmente, apófise tibial prolateral menos projetada e com ápice agudo, rádix afilado próximo à margem do címbio. Fêmea com epígino similar ao de A. janetae Miller, 2007 (Miller 2007, figs 124d, 128a, 131a, b) por compartilhar os ductos de copulação direcionados sobre as espermatecas e o átrio amplo e conspícuo (Fig. 7), diferencia-se pelos ductos de fertilização 

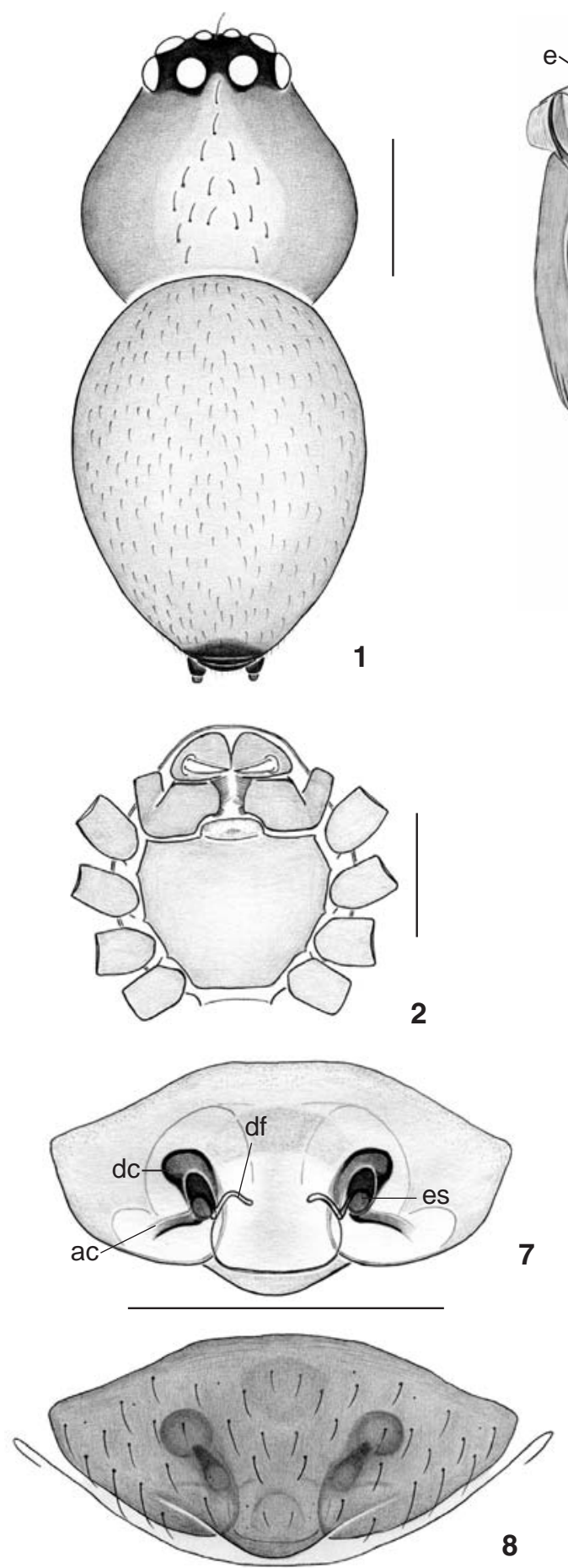
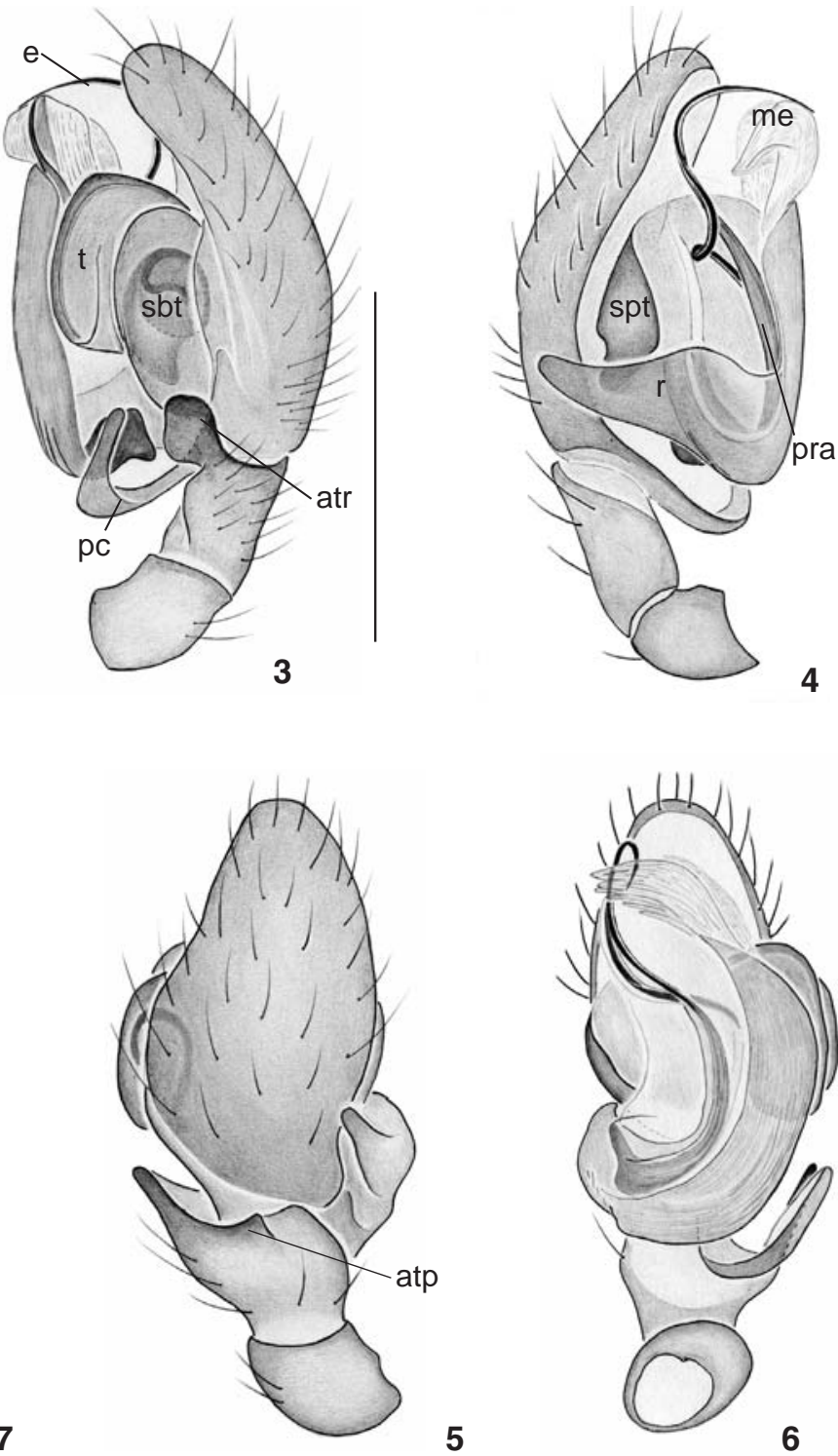

5

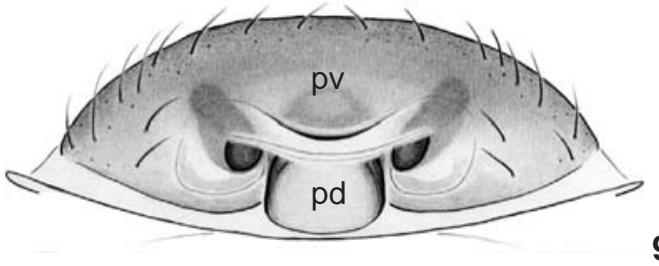

Figuras 1-9. Asemostera tacuapi sp. Nov.: (1) macho, corpo, vista dorsal; (2) esterno, vista ventral; (3-6) palpo do macho: (3) vista retrolateral; (4) vista prolateral; (5) vista dorsal; (6) vista ventral; (7-9) epígino: (7) vista dorsal, clarificado; (8) vista ventral; (9) vista posterior. (ac) Abertura de copulação, (atp) apófise tibial prolateral, (atr) apófise tibial retrolateral, (dc) ducto de copulação, (df) ducto de fertilização, (e) êmbolo, (es) espermateca, (me) membrana embólica, (pc) paracímbio, (pd) placa dorsal, (pra) processo radical anterior, (pv) placa ventral, ( $\mathrm{r}$ ) rádix, (sbt) subtégulo, (spt) supratégulo, (t) tégulo. Barras: 0,25 mm. 
mais distantes entre si e medianos, ductos de copulação não tão longos, aberturas de copulação próximas à margem posterior do epígino desembocando diretamente no átrio (Figs 7-9).

Descrição. Holótipo macho. Comprimento total 1,23. Carapaça, comprimento 0,55, largura 0,55, altura 0,32. Clípeo, altura 0,11. Esterno, comprimento 0,31, largura 0,33. Abdome, comprimento 0,78 , largura 0,57 , altura 0,57 . Fórmula das pernas $1 / 2 / 4 / 3$. Comprimento $1 / 2 / 3 / 4$ : fêmures $0,55 / 0,52 / 0,44 / 0,55$; patelas $0,20 / 0,20 / 0,15 / 0,17$; tíbias $0,40 / 0,37 / 0,27 / 0,40$; metatarsos 0,42/0,40/0,30/0,40; tarsos 0,37/0,37/0,15/0,27. Total 1,94/1,86/1,31/1,79. Posição da tricobótria no metatarso da perna I (TmI) 0,24. Metatarsos I-III com tricobótria dorsal, ausente no IV. Carapaça amarelo-clara, mais escura nas laterais. Região mediana com uma série de cerdas e uma fila disposta até a altura dos OMP. Clípeo amarelo-claro e glabro. Área ocular enegrecida, mais larga do que longa, com longas cerdas entre os OLP e OMP. Fila ocular anterior 0,23 e posterior 0,27. OLP apresentando o maior diâmetro $(0,08)$ e OMA, o menor $(0,03)$. Quelíceras amarelo-claras com quatro dentes na promargem e três na retromargem. Lábio muito piloso. Esterno amarelo-claro, truncado posteriormente (Fig. 2). Enditos amarelo-claros. Pernas com coxas amarelo-claras, ampla distância entre o par de coxas IV. Artículos das pernas amarelo-claros, exceto metatarso e tarso mais escuros. Abdômen oval (Fig. 1), com dorso amareloclaro, amplamente coberto por pêlos curtos; posteriormente, com mancha negra terminal cobrindo o tubérculo anal. Fiandeiras laterais anteriores e médias negras, fiandeiras laterais posteriores amareladas. Colulo amarelo-claro, densamente piloso. Palpo com êmbolo filiforme, longo, com porção terminal junto à membrana embólica (Figs 3, 4 e 6), apófise tibial retrolateral projetada e esclerotinizada distalmente (Fig. 3), apófise tibial prolateral pouco desenvolvida (Fig. 5) e rádix estreito, cujo ápice ultrapassa a borda do címbio (Fig. 4).

Parátipo fêmea (Triunfo, MCN 42741). Comprimento total 1,62. Carapaça, comprimento 0,57, largura 0,55, altura 0,26. Clípeo, altura 0,08. Esterno, comprimento 0,35, largura 0,44. Abdome, comprimento 1,20, largura 1,03, altura 0,80. Fórmula das pernas $1 / 2 / 4 / 3$. Comprimento $1 / 2 / 3 / 4$ : fêmures $0,62 / 0,62$ / $0,47 / 0,62$; patelas $0,18 / 0,18 / 0,17 / 0,20$; tíbias $0,50 / 0,47 / 0,35 /$ 0,42; metatarsos 0,47/0,44/0,42/0,47; tarsos 0,38/0,40/0,32/0,36. Total 2,15/2,11/1,73/2,07. Posição da tricobótria no metatarso da perna I (TmI) 0,30. Metatarsos com tricobótria dorsal, exceto o IV. Carapaça amarela, tonalidade mais escura do que no macho. Região mediana com várias cerdas e uma fila disposta até os OMP. Clípeo amarelo e glabro. Área ocular enegrecida, exceto entre os OMP, mais larga do que longa. Fila ocular anterior 0,25 e posterior 0,27. OLP apresentando o maior diâmetro $(0,07)$ e OMA, o menor $(0,03)$. Quelíceras amarelo-claras, promargem com quatro dentes e retromargem com três. Lábio castanho-escuro amplamente piloso. Esterno amarelo com bordas castanhoamareladas, truncado posteriormente. Enditos amarelos. Pernas amarelas, com coxas IV bem distantes entre si. Pedipalpo com tarso longo e intumescido. Abdômen oval, dorsalmente amare- lo-claro, densamente revestido por pêlos curtos; posteriormente, com mancha negra terminal cobrindo o tubérculo anal. Fiandeiras laterais anteriores e médias negras, laterais posteriores amarelas. Colulo densamente piloso, amarelado. Epígino com ductos de copulação curtos e com trajeto simples, direcionado sobre as espermatecas, aberturas de copulação localizadas no átrio, espermatecas alongadas e ductos de fertilização medianos, pouco esclerotinizados, originando-se na porção posterior das espermatecas (Figs 7-9).

Material examinado. BrasiL, Rio Grande do Sul: Canela (Usina Hidrelétrica Canastra), macho, 28.IX.2000, M. A.L. Marques leg. (MCN 33357); Montenegro, fêmea, 13.IX.2004, R.M. Morais leg. (MCN 42195); fêmea, 07.VII.1977, E.H. Buckup leg. (MCN 6162); Triunfo (Parque Copesul de Proteção Ambiental), fêmea, 18.IX.2006, R. Ott \& A. Barcellos leg. (MCN 42163); 10 machos, 3 fêmeas, 25.I.1990, 11.IX.1992, 24.IX.1999, M. A. L. Marques leg. (MCN 19395, 22382, 43326); fêmea, 28.VIII.2007, E.N.L. Rodrigues leg. (MCN 43497).

Distribuição. Sul do Brasil (Rio Grande do Sul).

\section{Asemostera latithorax (Keyserling, 1886)}

Erigone latithorax Keyserling, 1886: 274-275, fig. 324, holótipo macho, Blumenau, Santa Catarina, Brasil, depositado no BMNH, não examinado; Keyserling, 1891: 244.

Asemostera latithorax; Simon, 1898: 269-270, figs 268-271; Petrunkevitch, 1911: 529; 1928: 92; Mello-Leitão, 1927: 389; Roewer, 1954b: 1715; Roth, 1965: 291-292; Platnick, 2007; Miller, 2007: 168-171, figs 122, 124f, 126e, f, i, 128d. Agelenopsis latithorax; Roewer, 1954a: 43. Lapsus calami.

Pelidida albida Simon, 1898: 270, síntipos machos, 2 fêmeas, Serra do Caraça, Catas Altas, Minas Gerais, Brasil, depositado no MNHN, não examinado; Petrunkevitch, 1911: 535; 1928: 93; Roewer, 1954a: 69; Bonnet, 1958: 3455; Roth, 1965: 292; Miller, 2007: 169; Platnick, 2007.

Novo registro. BrasiL, Rio Grande do Sul: Canela (Passo dos Bugres, Usina Hidrelétrica Canastra), 2 fêmeas, 27.IX.2000, M.A.L. Marques leg. (MCN 33316); São Francisco de Paula (Barragem dos Bugres), 2 machos, 18.XI.1997, M.A.L. Marques leg. (MCN 28758).

Distribuição. Brasil, região Sudeste (Minas Gerais e Rio de Janeiro) e Sul (Santa Catarina e Rio Grande do Sul).

\section{AGRADECIMENTOS}

À curadora da coleção aracnológica do Museu de Ciências Naturais (MCN) Erica H. Buckup, pelo acesso ao material e à bibliografia. À direção do MCN pelo uso das dependências e equipamentos do museu. A Jeremy A. Miller pelo envio de bibliografia. Aos revisores anônimos pelas sugestões apresentadas. Ao CNPq pela concessão da bolsa de doutorado.

\section{REFERÊNCIAS BIBLIOGRÁFICAS}

Bonnet, P. 1958. Bibliographia Araneorum. Volume 2, part 4, N-S. Toulouse, Les Frères Douladoure, 3027-4230p. 
Keyserling, E. 1886. Die Spinnen Amerikas: Theridiidae. Nürnberg, vol. 2, part 2, 295p.

Keyserling, E. 1891. Die Spinnen Amerikas: Brasilianische Spinnen. Nürnberg, vol. 3, 278p.

Mello-Leitão, C.F. 1927. Arachnideos de Santa Catharina (Brasil). Revista do Museu Paulista 15: 393-418.

Miller, J.A. 2007. Review of Erigonine Spider Genera in the Neotropics (Araneae: Linyphiidae, Erigoninae). Zoological Journal of the Linnean Society 149 (Suppl. 1): 1-263.

Millidge, A.F. 1980. The erigonine spiders of North America. Part 1. Introduction and taxonomic background (Araneae: Linyphiidae). The Journal of Arachnology 8: 97-107.

Petrunkevitch, A. 1911. A synonymic index-catalogue of spiders of North, Central and South America with all adjacent islands, Greenland, Bermuda, West Indies, Terra del Fuego, Galapagos, etc. Bulletin of the American Museum of Natural History 29: 1-791.
Petrunkevitch, A. 1928. Systema aranearum. Transactions of the Connecticut Academy of Arts and Sciences 29: 1-270.

Platnick, N.I. 2007. The world spider catalog, version 7.5. American Museum of Natural History. Available on line at: http://research.amnh.org/entomology/spiders/catalog/ index.html [Accessed in 16/V/2007]

Roewer, C.F. 1954a. Katalog der Araneae von 1758 bis 1940. Bruxelles, Institut Royale des Sciences Naturelles, vol. 2, Abt. A, p. 1-924p.

Roewer, C.F. 1954b. Katalog der Araneae von 1758 bis 1940. Bruxelles, Institut Royale des Sciences Naturelles, vol. 2, Abt. B, p. 925-1751.

Rотн, V. 1965. Genera erroneously placed in the spider families Agelenidae and Pisauridae (Araneida: Arachnida). Annals of the Entomological Society of America 58: 289-292.

SIMON, E. 1898. Histoire Naturelle des Araignées. Paris, Librarie Encyclopédique de Roret, vol. 2, p. 193-380.

Recebido em 29.V.2007; aceito em 12.XI.2007. 